\title{
NONSTATIONARY TEMPERATURE FIELDS IN AN ISOTROPIC HALF-SPACE UNDER MIXED BOUNDARY CONDITIONS CHARACTERISTIC OF TECHNOLOGIES OF LASER THERAPY IN MEDICINE
}

\author{
V. P. Kozlov and P. A. Mandrik
}

UDC 517.968,536.24

For the first time, the solution of the heat-conduction equation with mixed boundary conditions (BCs) is obtained as applied to the model of an isotropic nontransparent half-space, whose surface $z=0$ is heated through the circular region of radius $r=R$ with a heat-flux density characteristic of a laser heat source, while outside of the circle of $r>R$ and $z=0$ there occurs intense cooling at the value of the heat-transfer coefficient $\alpha=\infty$.

The process of heating of biological objects by means of laser radiation sources is used in medical clinics for treatment, for example, of eye diseases. Therefore, to organize safe laser therapy, it is very important to know the laws of the development of spatial temperature fields in the objects investigated with the aim of limiting them to safe temperatures (a direct problem of mathematical physics). On the other hand, the developed temperature fields are a result of the local heating of the surface by a laser source with the corresponding heat-flux density $q(r, t)$ through the circular region on this surface. The determination of the law of change in the specific heat flux $q(r, t)$, generated by the laser source, at which the temperature of the irradiated surface does not exceed admissibie values for biological objects is an urgent and practically important problem (an inverse problem of mathematical physics).

It should be noted that the heating of an isotropic half-space through the circular region under unmixed BCs has been studied in [1-4], which are associated with the corresponding solutions of Neumann problems. In the case of mixed BCs, the body of mathematics for seeking solutions of nonstationary heat-conduction problems is considerably complicated, and the solutions themselves cannot be obtained in a closed analytical form.

Let us dwell on the physical aspect of the subject of investigation that affects the mathematical statement of this problem. Since 1958, when A. L. Shavlov and Ch. G. Tauns [5] assumed that the principle of light amplification due to forced radiation can be extended to the optical spectrum, laser technology has achieved considerable advances. Different types of lasers have been created: gas, solid-state, semiconductor, dye lasers, and others, which are used in various fields of science, engineering, technology, and medicine. In most lasers, a beam of diameter $2 R$ possesses azimuthal symmetry in the cross section with the highest intensity on the axis $r=0$. As the distance $r$ from the beam axis increases, the intensity of the radiation density (of the specific heat flux) $q(r, \tau)$ changes following the exponential law

$$
q(r, \tau)=q_{0}(\tau) \exp \left(-\frac{r^{2}}{R^{2}}\right),
$$

where $\tau$ is the time, $r$ is the running cylindrical coordinate, $R$ is the radius of the radiation beam in its cross section, and $q(r, \tau)$ is the heat-flux density.

The value of $r$ at which the radiation intensity decreases $e$ times compared to the intensity on the beam axis $r=0$ is called the transverse dimension $R$ of the beam [4]. Generally speaking, $R$ changes from point to point along the beam axis. Hereafter, in the mathematical statement of our problem we will assume that a

Belarusian State University, Minsk, Belarus. Translated from Inzhenerno-Fizicheskii Zhurnal, Vol. 73, No. 3, pp. 637-644, May-June, 2000. Original article submitted November 16, 1999. 
beam of diameter $2 R$ is focused onto the surface $(z=0)$ (normally to the surface) of the half-space, while the intensity of the beam on the surface of the object investigated in the circular region $0<r<R$ changes following law (1) with account for the absorption capacity of the surface.

We recall that the ratio of the radiation flux absorbed by a given body to that incident on the body is called the absorption capacity. Therefore, the second-kind boundary condition on a part of the surface $(z=0$, $0<r<R$ ) will be written in the form

$$
-\lambda \theta_{z}(r, 0, \tau)=\alpha_{\mathrm{s}} q_{0}(\tau) \exp \left(-\frac{r^{2}}{R^{2}}\right), \quad 0<r<R,
$$

where $\alpha_{\mathrm{S}}$ is the coefficient of absorption capacity for the surface of the nontransparent isotropic half-space $\left(0<\alpha_{\mathrm{s}} \leq 1\right), q_{0}(r)$ is the time law of change in the specific power of laser radiation, and $\lambda>0$ is the thermal conductivity coefficient of the object under investigation.

Thus, it is necessary to solve the equation

$$
\theta_{r r}(r, z, \tau)+r^{-1} \theta(r, z, \tau)+\theta_{z z}(r, z, \tau)=a^{-1} \theta_{\tau}(r, z, \tau), r, z, \tau>0,
$$

(where $a>0$ is the thermal diffusivity coefficient of the object, $r$ and $z>0$ are the cylindrical coordinates, and $\tau>0$ is the time) under the mixed discontinuous boundary conditions

$$
\begin{gathered}
-\theta_{\Sigma}(r, 0, \tau)=\frac{\alpha_{\mathrm{s}}}{\lambda} q_{0}(\tau) \exp \left(-\frac{r^{2}}{R^{2}}\right), \quad 0<r<R ; \\
\theta(r, 0, \tau)=0, \quad r<R<\infty,
\end{gathered}
$$

and the initial condition $\theta(r, z, 0)=0$.

Applying the integral Laplace transform to Eqs. (3)-(5)

$$
L[\theta(r, z, \tau)]=\bar{\theta}(r, z, s)=\int_{0}^{\infty} \exp (-s \tau) \theta(r, z, \tau) d \tau
$$

(where $\operatorname{Re} s>0$ and for brevity of statements is implied further by default) and taking into account the condition of limitation $\theta(r, z, \tau)$ with $\sqrt{r^{2}+z^{2}} \rightarrow \infty$, it is easy to obtain a solution for representation of $\bar{\theta}(r, z, s)$ in the form [6-8]

$$
\bar{\theta}(r, z, s)=\int_{0}^{\infty} \bar{C}^{*}(p, s) \exp \left(-z \sqrt{ }\left(p^{2}+\frac{s}{a}\right)\right) J_{0}(p r) d p,
$$

where $\bar{C}^{*}(p, s)$ is the unknown function.

Mixed boundary conditions (4) and (5) for representation (7) take the form

$$
\begin{gathered}
-\bar{\theta}_{z}(r, 0, s)=\frac{\alpha_{s}}{\lambda} \bar{q}_{0}(s) \exp \left\{-\frac{r^{2}}{R^{2}}\right\}, 0<r<R, \\
\bar{\theta}(r, 0, s)=0, \quad r<R<\infty,
\end{gathered}
$$

where 


$$
\bar{q}_{0}(s)=\int_{0}^{\infty} \exp (-s \tau) q_{0}(\tau) d \tau .
$$

Thus, taking into account mixed boundary conditions (4) and (5), we reduce the solution of differential problem (3)-(5) to the solution of the following paired integral equations in the region of $L$-transforms relative to the value of $\bar{C}^{*}(p, s)$ :

$$
\begin{aligned}
\int_{0}^{\infty} \bar{C}^{*}(p, s) & \sqrt{ }\left(p^{2}+\frac{s}{a}\right) J_{0}(p r) d p=\bar{q}_{0}^{*}(s) \exp \left(-\frac{r^{2}}{R^{2}}\right), 0<r<R, \\
& \int_{0}^{\infty} \bar{C}^{*}(p, s) J_{0}(p r) d p=0, \quad R<r<\infty,
\end{aligned}
$$

where $J_{0}(p r)$ is the Bessel function of the real argument $[3,9]$ and $\bar{q}_{0}^{*}(s)=\frac{\alpha_{s^{-}}}{\lambda} q_{0}(s)$.

To solve Eqs. (8) and (9), we introduce into consideration another analytical function $\bar{\varphi}^{*}(t, s)$ by means of the relation $[6,7]$

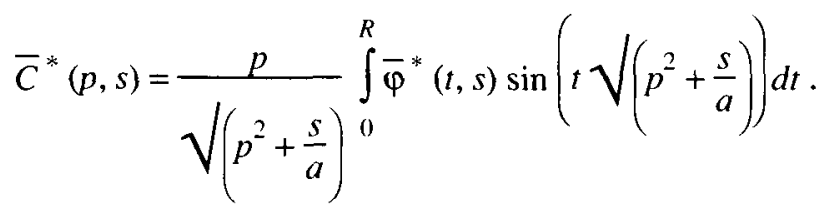

Substitution of Eq. (10) into Eq. (9) provides immediately the fulfillment of the second paired equation due to the value of the following discontinuous integral $[9, \mathrm{p} .203]$ for $r>R$ :

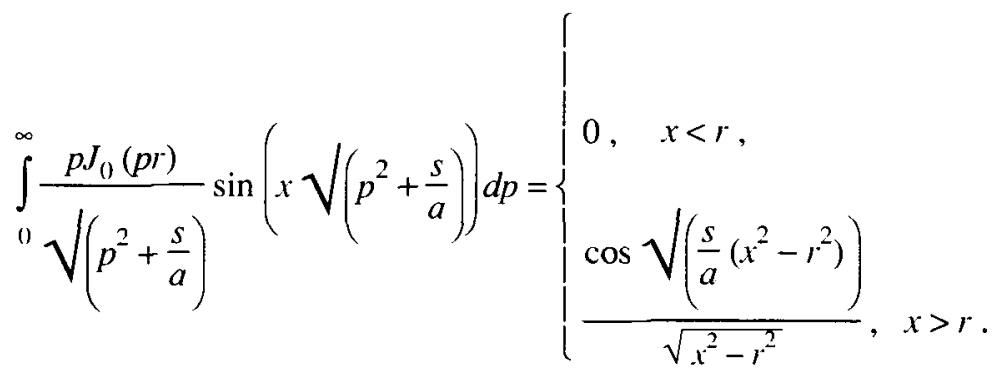

Substituting further Eq. (10) into the first paired equation (8), we obtain the integral equation with the $L$-parameter for determining $\bar{\varphi}^{*}(t, s)$ :

$$
\begin{aligned}
& \int_{0}^{r} \frac{t \bar{\varphi}^{*}(t, s)}{\sqrt{r^{2}-t^{2}}} \exp \left(-\sqrt{ }\left(\frac{s}{a}\left(r^{2}-t^{2}\right)\right)\right) d t+\int_{0}^{R} \bar{\varphi}^{*}(t, s) \sin \left(t \sqrt{ }\left(\frac{s}{a}\right)\right) d t- \\
& -\int_{r}^{R} \frac{t \bar{\varphi}^{*}(t, s)}{\sqrt{t^{2}-r^{2}}} \sin \left(\sqrt{ }\left(\frac{s}{a}\left(t^{2}-r^{2}\right)\right)\right) d t=\bar{q}_{0}^{*}(s) \int_{0}^{r} \exp \left(-\frac{\rho^{2}}{R^{2}}\right) \rho d \rho, 0<r<R .
\end{aligned}
$$

We note that in deriving Eq. (11) the following formulas [9] were used: 


$$
\begin{aligned}
& p r J_{0}(p r)=\frac{d}{d r}\left[r J_{1}(p r)\right],
\end{aligned}
$$

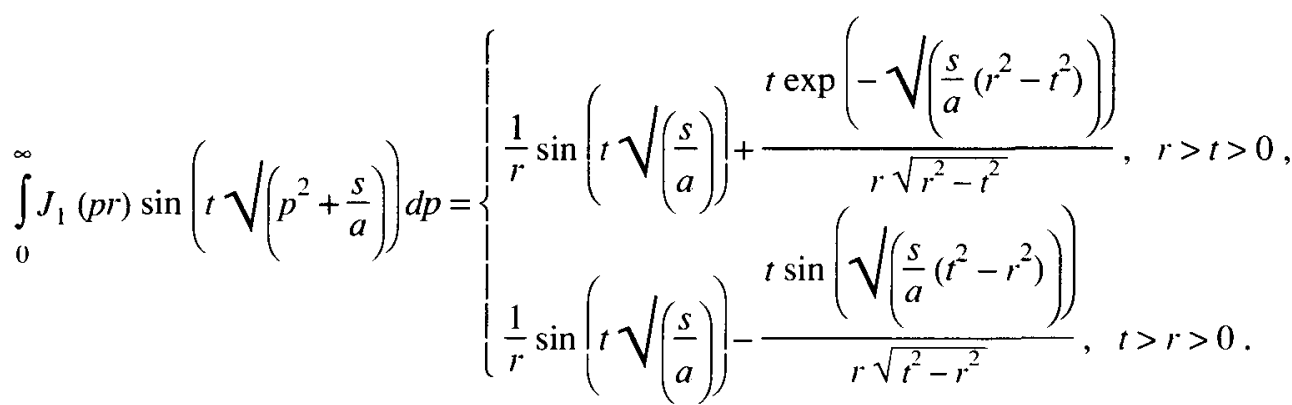

Next, to determine $\bar{\varphi}^{*}(t, s)$ from Eq. (11), we proceed in a way which differs from that described in [7], i.e., we reduce Eq. (11) to an equation similar in appearance to a Fredholm integral equation but with the parameter $s$. For this, we multiply the left- and right-hand sides of equality (11) by the integrating factor $\int_{0}^{r} \frac{2 \cos \left(\frac{s}{a}\left(r^{2}-\mu^{2}\right)\right)^{1 / 2}}{\sqrt{r^{2}-\mu^{2}}} \mu$, having preliminarily replaced $r$ by $\mu$ in Eq. (11). Then Eq. (11) takes the form

$$
\begin{aligned}
& \int_{0}^{r} \frac{2 \cos \left(\sqrt{\left.\left(\frac{s}{a}\left(r^{2}-\mu^{2}\right)\right)\right) \mu}\right.}{\sqrt{r^{2}-\mu^{2}}} \int_{0}^{\mu} \frac{t \bar{\varphi}^{*}(t, s) \exp \left(-\sqrt{ }\left(\frac{s}{a}\left(\mu^{2}-t^{2}\right)\right)\right.}{\sqrt{\mu^{2}-t^{2}}} d t d \mu+ \\
& +\int_{0}^{r} \frac{2 \cos \left(\sqrt{ }\left(\frac{s}{a}\left(r^{2}-\mu^{2}\right)\right)\right) \mu}{\sqrt{r^{2}-\mu^{2}}} \int_{0}^{R} \bar{\varphi}^{*}(t, s) \sin \left(t \sqrt{ }\left(\frac{s}{a}\right)\right) d t d \mu- \\
& -\int_{0}^{r} \frac{2 \cos \left(\sqrt{ }\left(\frac{s}{a}\left(r^{2}-\mu^{2}\right)\right)\right) \mu R_{\mu} t \bar{\varphi}^{*}(t, s) \sin \left(\sqrt{ }\left(\frac{s}{a}\left(t^{2}-\mu^{2}\right)\right)\right.}{\sqrt{r^{2}-\mu^{2}}} d t d \mu= \\
& =\bar{q}_{0}^{*}(s) \int_{0}^{r} \frac{2 \cos \left(\sqrt{ }\left(\frac{s}{a}\left(r^{2}-\mu^{2}\right)\right)\right) \mu}{\sqrt{r^{2}-\mu^{2}}} \int_{0}^{\mu} \exp \left(-\frac{\rho^{2}}{R^{2}}\right) \rho d \rho d \mu, 0<r<R .
\end{aligned}
$$

Changing in Eq. (12) the order of integration by the known Dirichlet formula for iterated integrals, we obtain in the right-hand side of Eq. (12)

$$
\bar{q}_{0}^{*}(s) \int_{0}^{r} \frac{2 \cos \left(\sqrt{ }\left(\frac{s}{a}\left(r^{2}-\mu^{2}\right)\right) \mu \mu\right.}{\sqrt{r^{2}-\mu^{2}}} \int_{0}^{\mu} \exp \left(-\frac{\rho^{2}}{R^{2}}\right) \rho d \rho d \mu=
$$




$$
\begin{gathered}
=\bar{q}_{0}^{*}(s) \int_{0}^{r} \exp \left(-\frac{\rho^{2}}{R^{2}}\right) \rho d \rho \int_{\rho}^{r} \frac{2 \cos \left(\sqrt{\left(\frac{s}{a}\left(r^{2}-\mu^{2}\right)\right)}\right) \mu}{\sqrt{r^{2}-\mu^{2}}} d \mu= \\
=\frac{2 \bar{q}_{0}^{*}(s)}{\sqrt{ }\left(\frac{s}{a}\right)} \int_{0}^{r} \exp \left(-\frac{\rho^{2}}{R^{2}}\right) \sin \left(\sqrt{ }\left(\frac{s}{a}\left(r^{2}-\rho^{2}\right)\right)\right) \rho d \rho, 0<r<R .
\end{gathered}
$$

Acting in a similar way in the left-hand side of Eq. (12), we arrive at the equation

$$
\begin{gathered}
\pi \int_{0}^{r} t \bar{\varphi}^{*}(t, s) d t+\sqrt{\left(\frac{a}{s}\right)} \int_{0}^{R} 2 \bar{\varphi}^{*}(t, s) \sin \left(t \sqrt{ }\left(\frac{s}{a}\right)\right) \sin \left(r \sqrt{ }\left(\frac{s}{a}\right)\right) d t- \\
\quad-\int_{0}^{R} t \bar{\varphi}^{*}(t, s)\left[\operatorname{Si}\left(\sqrt{ }\left(\frac{s}{a}\right)(t+r)\right)+\operatorname{Si}\left(\sqrt{ }\left(\frac{s}{a}\right)(r-t)\right)\right] d t= \\
=2 \bar{q}_{0}^{*}(s) \sqrt{\left(\frac{a}{s}\right)} \int_{0}^{r} \exp \left(-\frac{\rho^{2}}{R^{2}}\right) \sin \left(\sqrt{ }\left(\frac{s}{a}\left(r^{2}-\rho^{2}\right)\right)\right) \rho d \rho, 0<r<R .
\end{gathered}
$$

Next, differentiating the last equality with respect to $r$, we obtain

$$
\begin{gathered}
\bar{\varphi}^{*}(r, s)-\frac{1}{\pi} \int_{0}^{R} \bar{\varphi}^{*}(t, s)\left[\frac{\sin \left(\sqrt{ }\left(\frac{s}{a}(t-r)\right)\right)}{t-r}-\frac{\sin \left(\sqrt{ }\left(\frac{s}{a}(t+r)\right)\right)}{t+r}\right] d t= \\
=\frac{2}{\pi} \bar{q}_{0}^{*}(s) \int_{0}^{r} \exp \left(-\frac{\rho^{2}}{R^{2}}\right) \frac{\cos \left(\sqrt{\left(\frac{s}{a}\left(r^{2}-\rho^{2}\right)\right)}\right.}{\sqrt{r^{2}-\rho^{2}}} \rho d \rho, 0<r<R .
\end{gathered}
$$

The integral equation with the $L$-parameter (13) is the initial one for determining the analytical function $\bar{\varphi}^{*}(r, s)$. In [8], the solution of Eq. (13) was obtained for a constant right-hand side, in which the function assigned in the circle $(0<r<R, z=0)$ was equal to $\frac{2 q_{0}}{\pi \lambda s \sqrt{s / a}} \sin (r \sqrt{s / a})$. We introduce into Eq. (13) the notation $\bar{\psi}(r, s)=\frac{\bar{\varphi}^{*}(r, s)}{s \bar{q}_{0}^{*}(s)}$, where $\bar{q}_{0}^{*}(s) \neq 0$ and $\bar{K}_{2}(r, t, s)=\frac{\sin (\sqrt{s / a}(t-r))}{t-r}-\frac{\sin (\sqrt{s / a}(t+r))}{t+r}$, at which Eq. (13) will be written as follows:

$$
\bar{\psi}(r, s)=\frac{2}{\pi s} \int_{0}^{r} \exp \left(-\frac{\rho^{2}}{R^{2}}\right) \frac{\cos \left(\sqrt{\left(\frac{s}{a}\left(r^{2}-\rho^{2}\right)\right)}\right)}{\sqrt{r^{2}-\rho^{2}}} \rho d \rho+
$$




$$
+\frac{1}{\pi} \int_{0}^{R} \bar{\psi}(t, s) \bar{K}_{2}(r, t, s) d t, \quad 0<r<R .
$$

We will seek a solution of Eq. (14) in the form of a series of functions

$$
\bar{\psi}(r, s)=\frac{1}{s} \exp \left(-R \sqrt{\left.\left(\frac{s}{a}\right)\right)} \sum_{n=1}^{\infty} \psi_{n}(r)(\sqrt{s})^{n},\right.
$$

and then we substitute this series into Eq. (14) and, as a result, obtain the equality

$$
\begin{gathered}
\sum_{n=0}^{\infty} \Psi_{n}(r)(\sqrt{s})^{n-2}=\frac{2}{\pi s} \int_{0}^{r} \frac{\exp \left(-\frac{\rho^{2}}{R^{2}}\right)}{\sqrt{r^{2}-\rho^{2}}} \rho \exp \left(R \sqrt{ }\left(\frac{s}{a}\right)\right) \cos \left(\sqrt{ }\left(\frac{s}{a}\left(r^{2}-\rho^{2}\right)\right)\right) d p+ \\
+\frac{1}{\pi s} \int_{0}^{R} \bar{K}_{2}(r, t, s) \sum_{n=1}^{\infty} \Psi_{n}(t)(\sqrt{s})^{n} d t, 0<r<R .
\end{gathered}
$$

As a power series, we can write the following product:

$$
\begin{aligned}
& \exp \left(R \sqrt{\left.\left(\frac{s}{a}\right)\right)} \cos \left(\sqrt{ }\left(\frac{s}{a}\left(r^{2}-\rho^{2}\right)\right)\right)=\right. \\
& =\sum_{n=0}^{\infty}(\sqrt{s})^{n}\left[\frac{1}{2 n !}\left(\frac{1}{\sqrt{a}}\right)^{n}\left[\left(R+i \sqrt{r^{2}-\rho^{2}}\right)^{n}+\left(R-i \sqrt{r^{2}-\rho^{2}}\right)^{n}\right]\right]= \\
& =\sum_{n=0}^{\infty}(\sqrt{s})^{n} \sum_{j=0}^{n} A_{n, j}^{*}(R)\left(\sqrt{r^{2}-\rho^{2}}\right)^{j}, \quad r>\rho,
\end{aligned}
$$

where

$$
A_{n, j}^{*}(R)=\left(\begin{array}{l}
n \\
j
\end{array}\right) \frac{1}{n !(\sqrt{a})^{n}} R^{n-j} \cos \left(\frac{j \pi}{2}\right),
$$

$\left(\begin{array}{l}n \\ j\end{array}\right)$ are the binomial coefficients.

The following product can easily be written in the Cauchy form [10]:

$$
\begin{aligned}
& \bar{K}_{2}(r, t, s) \sum_{n=0}^{\infty} \Psi_{n}(t)(\sqrt{s})^{n}=\sum_{n=0}^{\infty} C_{m}^{*}(t, r)(\sqrt{s})^{m} \sum_{n=0}^{\infty} \Psi_{n}(t)(\sqrt{s})^{n}= \\
& =\sum_{n=0}^{\infty}(\sqrt{s})^{n} \sum_{k=0}^{n} \Psi_{k}(t) C_{n-k}^{*}(t, r)=\sum_{n=0}^{\infty}(\sqrt{s})^{n} \sum_{m=0}^{n} C_{m}^{*}(t, r) \Psi_{n-m}(t),
\end{aligned}
$$

where $\psi_{k}(t)$ are the unknown functional coefficients of series (15), while $C_{m}^{*}(t, r)$ has the form 


$$
\begin{aligned}
& C_{m}^{*}(t, r)=\frac{1}{m !}\left(\frac{1}{\sqrt{a}}\right)^{m} \sin \left(\frac{m \pi}{2}\right)\left[(t-r)^{m-1}-(t+r)^{m-1}\right]= \\
& =\frac{1}{m !}\left(\frac{1}{\sqrt{a}}\right)^{m} \sin \left(\frac{m \pi}{2}\right)^{m-1} \sum_{j=0}^{m}\left(\begin{array}{c}
m-1 \\
j
\end{array}\right) t^{m-j-1}, r^{j}\left[(-1)^{j}-1\right] .
\end{aligned}
$$

Substituting series (17) and (18) into formula (16), we obtain an equation for determining the coefficients $\psi_{n}(r)$, which, as is easy to see, will not be the integral equation for the unknown function $\psi_{n}(t)$ (since $\left.C_{0}^{*}(t, r) \equiv 0\right)$ but will represent the recursion formula

$$
\begin{aligned}
\Psi_{n}(r)=\frac{2}{\pi} & \sum_{j=0}^{n} A_{n, j}^{*}(R) \int_{0}^{r} \exp \left(-\frac{\rho^{2}}{R^{2}}\right)\left(r^{2}-\rho^{2}\right)^{\frac{j-1}{2}} \rho d \rho+ \\
& +\frac{1}{\pi} \sum_{m=0}^{n} \int_{0}^{R} C_{m}^{*}(t, r) \Psi_{n-m}(t) d t,
\end{aligned}
$$

where $C_{m}^{*}(t, r) \equiv 0$ with $m=0,1,2,4,6,8, \ldots$.

Thus, for example, from Eq. (19) we obtain

$$
\begin{gathered}
\Psi_{0}(r)=\frac{R}{\sqrt{\pi}} \exp \left(-\frac{r^{2}}{R^{2}}\right) \operatorname{erfi}\left(\frac{r}{R}\right) ; \quad \Psi_{1}(r)=\frac{R^{2}}{\sqrt{\pi a}} \exp \left(-\frac{r^{2}}{R^{2}}\right) \operatorname{erfi}\left(\frac{r}{R}\right) ; \\
\Psi_{2}(r)=\frac{3 R^{3}}{4 a \sqrt{\pi}} \exp \left(-\frac{r^{2}}{R^{2}}\right) \operatorname{erfi}\left(\frac{r}{R}\right)-\frac{r R^{2}}{2 \pi a} \\
\Psi_{3}(r)=\frac{5 R^{4}}{12 \sqrt{\pi} a \sqrt{a}} \exp \left(-\frac{r^{2}}{R^{2}}\right) \operatorname{erfi}\left(\frac{r}{R}\right)- \\
-\frac{r R^{3}}{2 \pi a \sqrt{a}}+\frac{r R^{2}}{3 \pi^{2} a \sqrt{a}}\left[\sqrt{\pi} R \text { erfi }\left(\frac{r}{R}\right)+2 r\right] \text { etc. }
\end{gathered}
$$

where

$$
\operatorname{erfi}(x)=-i \operatorname{erf}(i x)=\frac{2}{\sqrt{\pi}} \int_{0}^{x} \exp \left(t^{2}\right) d t=\frac{2}{\sqrt{\pi}} \sum_{k=0}^{\infty} \frac{x^{2 k+1}}{k !(2 k+1)} .
$$

Substitution of $\psi_{n}(r)$ from Eq. (19) into Eq. (15) gives the representation

$$
\begin{gathered}
\bar{\psi}(r, s)=\frac{2}{\pi s} \exp \left(-R \sqrt{\left.\left(\frac{s}{a}\right)\right) \int_{0}^{r} \exp \left(-\frac{\rho^{2}}{R^{2}}\right)\left(r^{2}-\rho^{2}\right)^{-0.5} \rho d \rho+}\right. \\
+\frac{2}{\pi} \sum_{m=0}^{\infty} \exp \left(-R \sqrt{ }\left(\frac{s}{a}\right)\right) s^{\frac{m-1}{2}}\left[\sum_{j=0}^{m+1} A_{m+1, j}^{*}(R) \int_{0}^{r} \exp \left(-\frac{\rho^{2}}{R^{2}}\right)\left(r^{2}-\rho^{2}\right)^{\frac{j-1}{2}} \rho d \rho+\right.
\end{gathered}
$$




$$
\left.+\frac{1}{2} \sum_{k=0}^{m+1} \int_{0}^{R} C_{k}^{*}(t, r) \psi_{m-k+1}(t) d t\right], \quad 0<r<R
$$

for which there is the inverse Laplace transform [11], since

$$
\begin{gathered}
L^{-1}\left[\frac{1}{s} \exp (-k \sqrt{s})\right]=\operatorname{erfc}\left(\frac{k}{2 \sqrt{\tau}}\right), \\
L^{-1}\left[s^{\frac{j-1}{2}} \exp (-k \sqrt{s})\right]=\frac{\exp \left(-\frac{k^{2}}{4 \tau}\right)}{2^{j} \sqrt{\pi \tau^{j+1}}} H_{j}\left(\frac{k}{2 \sqrt{\tau}}\right),
\end{gathered}
$$

where

$$
H_{j}(x)=j ! \sum_{l=l)}^{\left[\frac{j}{2}\right]} \frac{(-1)^{l}}{l !(j-2 l) !}(2 x)^{j-2 l}
$$

is the Hermite polynomial $[3,9]$.

The value of $\bar{\varphi}^{*}(r, s)$ is found from the equation

$$
\bar{\varphi}^{*}(r, s)=s \bar{q}_{0}^{*}(s) \bar{\psi}(r, s),
$$

while for determination of the original $\varphi^{*}(r, \tau)$ at the known $L^{-1}\left[s \bar{q}_{0}^{*}(s)\right]=q_{0}^{*}(\tau)$ and $L^{-1}[\bar{\psi}(r, s)]=\psi(r, \tau)$ we have

$$
\varphi^{*}(r, \tau)=L^{-1}\left[\bar{\varphi}^{*}(r, s)\right]=L^{-1}\left[s \bar{q}_{0}^{*}(s) \bar{\psi}(r, s)\right]=\int_{0}^{\tau} \psi(r, \xi) q_{0}^{*}(\tau-\xi) d \xi
$$

We find the value of $\bar{C}^{*}(p, s)$ from Eq. (10) by substituting $\bar{\varphi}^{*}(t, s)$ into it. The temperature field for the representation of $\bar{\theta}(r, z, s)$ is determined from Eq. (7), and the original $\bar{\theta}(r, z, \tau)=L^{-1}[\bar{\theta}(r, z, s)]$ is found from the well-known [12] inversion formula.

In conclusion, it should be noted that, according to Eqs. (21) and (20), the stationary values of the corresponding functions with $s \rightarrow 0$ can be written in the form

$$
\begin{gathered}
\varphi^{*}(r)=\lim _{s \rightarrow 0}\left[s \bar{\varphi}^{*}(r, s)\right]=\frac{2}{\pi} \lim _{s \rightarrow 0}\left[s \bar{q}_{0}^{*}(s)\right] \int_{0}^{r} \exp \left(-\frac{\rho^{2}}{R^{2}}\right)\left(r^{2}-\rho^{2}\right)^{-(0.5} \rho d \rho, \\
\psi(r)=\lim _{s \rightarrow 0}[s \bar{\psi}(r, s)]=\frac{2}{\pi} \int_{0}^{r} \exp \left(-\frac{\rho^{2}}{R^{2}}\right) \frac{\rho}{\sqrt{r^{2}-\rho^{2}}} d \rho .
\end{gathered}
$$

Here the last formula coincides with the solution of the Laplace equation for a half-space $[13,14]$ with mixed boundary conditions

$$
-\theta_{z}(r, 0)=\frac{\alpha_{\mathrm{s}}}{\lambda} q_{0} \exp \left(-\frac{r^{2}}{R^{2}}\right), \quad 0<r<R ; \quad \theta(r, 0)=0, \quad r<R<\infty .
$$




\section{REFERENCES}

1. H. S. Carslaw and J. Jaeger, Conduction of Heat in Solids, Oxford Univ. Press (1959).

2. J. V. Beck, Int. J. Heat Mass Transfer, 24, 155-164 (1981).

3. V. P. Kozlov, Two-Dimensional Axially Symmetric Nonstationary Heat-Conduction Problems [in Russian], Minsk (1986).

4. V. P. Kozlov, Inzh.-Fiz. Zh., 54, No. 3, 484-493 (1988).

5. D. O'Shea, R. Collen, and U. Rods, Laser Technology [Russian translation], Moscow (1980).

6. N. A. Abdel'razak, Methods of Solving Two-Dimensional Problems of Nonstationary Heat-Conduction with Mixed and Unmixed Discontinuous Boundary Conditions. Author's Abstract of Candidate's Dissertation in Physical-Mathematical Sciences, Minsk (1996).

7. V. P. Kozlov, N. I. Yurchuk, and P. A. Mandrik, Inzh.-Fiz. Zh, 71, No. 4, 734-743 (1998).

8. V. P. Kozlov, N. I. Yurchuk, and P. A. Mandrik, Vestn. Belorus. Univ., Ser. 1, No. 2, $37-42$ (1999).

9. A. P. Prudnikov, Yu. A. Brychkov, and O. I. Marichev, Integrals and Series. Special Functions [in Russian], Moscow (1983).

10. G. M. Fikhtengol'ts, A Course in Differential and Integral Calculus [in Russian], Vol. 2, Moscow (1966).

11. M. Abramovits and I. Stigan (eds.), Handbook of Special Functions [Russian translation], Moscow (1979).

12. G. Bateman and A. Erdélyi, Tables of Integral Transformations, Vol. 1. Fourier, Laplace, and Mellin Transforms [in Russian], Moscow (1969).

13. Ya. S. Uflyand, Method of Paired Equations in Mathematical Physics Problems [in Russian], Leningrad (1977).

14. I. Sneddon, Mixed Boundary-Value Problems in Potential Theory, Amsterdam (1966). 